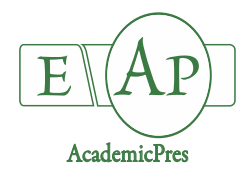

Zhou Y et al. (2020)
Notulae Botanicae Horti Agrobotanici Cluj-Napoca 48(4):1832-1844
DOI: $10.15835 /$ nbha48411960
Research Article

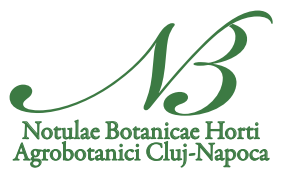

\title{
Molecular cloning and characterization of a lipoxygenase gene (CsLOX1) from cucumber
}

\author{
Yong ZHOU ${ }^{1}$, Mingyuan XU², Wei LAI ${ }^{2}$, Lianghai $\mathrm{CHEN}^{1}$, \\ Yingui YANG ${ }^{2}$, Shiqiang LIU ${ }^{1 *}$
}

\author{
${ }^{1}$ Jiangxi Agricultural University, College of Bioscience and Bioengineering, Nanchang 330045, \\ China; yongzhou@jxau.edu.cn; 15870644120@163.com; Isq_hn306@163.com (*corresponding author) \\ 2Jiangxi Agricultural University, College of Agronomy, Nanchang330045, China; $15797794879 @ 163 . c o m$; \\ 15797631915@163.com; yangyingui@163.com
}

\begin{abstract}
Lipoxygenases (LOXs) are non-heme iron enzymes that play crucial roles in many developmental processes during plant life, and defense responses against biotic and abiotic stresses. In this study, a lipoxygenase gene (CsLOXI) was cloned and characterized from cucumber (Cucumis sativus). The coding sequence (CDS) of $C S L O X 1$ was $741 \mathrm{bp}$, and encoded an 878 amino-acid residue protein, which was predicted to be located in the cytoplasm. CsLOX1 contained the conserved LH2/PLAT and lipoxygenase domains, as well as the representative 38 amino acids motif [His-(X)4-His-(X)4-His-(X)17-His-(X)8-His]. Multiple sequence alignment and phylogenetic analysis indicated that CsLOX1 was closely related to other dicot 9-LOXs and posesess the essential conserved residues involved in the binding of the iron atom. Promoter analysis suggested that several development-, stress-, and hormone-related cis-acting regulatory elements were present in the promoter region of CsLOX1. The function of CsLOX1 was assessed by overexpression it in Arabidopsis, and the transgenic plants were male sterile and displayed obviously increased floral shoots. These results provide some clues for revealing the biological roles of $C s L O X 1$ in cucumber.
\end{abstract}

Keywords: cucumber; expression pattern; lipoxygenase (LOX); transgenic Arabidopsis

\section{Introduction}

Lipoxygenase (LOX, EC 1.13.11.12) is a group of non-heme iron enzymes widely distributed in plants, animals, fungi, and bacteria (Yamamoto, 1992; Porta and Rocha-Sosa, 2001; Feussner and Wasternack, 2002; Brodhun and Feussner, 2011). In plants, LOXs can insert molecular oxygen into position carbon atom 9 or position carbon atom 13 of the oxygenation of polyunsaturated fatty acids (PUFAs) to produce 9hydroperoxide octadecadi(tri)enoic acids (9-HPOD/T) and 13-hydroperoxide octadecatrienoic acid (13HPOT) hydroperoxides, respectively, and then initiate the oxylipin biosynthesis to form a large class of biologically active compounds, such as jasmonic acid (JA), green leaf volatiles (GLVs) and other related compounds (Zhu et al., 2018; Zhou et al., 2019a; Viswanath et al., 2020). Therefore, plant LOXs can be classified into two groups: 9-LOXs and 13-LOXs, based on the carbon atom positional specificity of substrates 
( Feussner and Wasternack, 2002; Wasternack and Feussner, 2018). Beside 9-LOXs and 13-LOXs, plant LOXs displayed a dual 9/13-LOX specificity were also identified (Wang et al., 2008; Liu and Han, 2010; Padilla et al., 2012; Zhu et al., 2018). Furthermore, 13-LOXs can be further divided into two subgroups, type I 13-LOX and type II 13-LOX, according to their primary structure and sequence similarity ( Feussner and Wasternack, 2002; Liavonchanka and Feussner, 2006).

LOXs are encoded by a multi gene family in numerous plants, and $L O X$ family genes were identified in various plant species, such as Arabidopsis (6 genes) (Bannenberg et al., 2009; Umate, 2011), pepper (8 genes) (Sarde et al., 2018), radish (11 genes) (Wang et al., 2019), peach (12 genes) (Guo et al., 2017), rice (14 genes) (Umate, 2011), tomato (14 genes) (Upadhyay and Mattoo, 2018), apple (23 genes) (Vogt et al., 2013), and pear (23 genes) (Li et al., 2014). And functional characterizations of $L O X$ genes indicated that they play vital roles in a variety of developmental processes during plant life, such as tuber development (Kolomiets et al., 2001), nodule development (Hayashi et al., 2008), sex determination (Acosta et al., 2009), anther and pollen development (Caldelari et al., 2011), seed germination and longevity (Huang et al., 2014), leaf senescence (Hou et al., 2015; Springer et al., 2016), and fruit ripening ( Guo et al., 2017; Zhang et al., 2017), Moreover, LOXs also play key roles in defense responses against biotic and abiotic environmental stresses. For example, overexpression of TomLOXD (SILOXD) in transgenic tomato plants increased plant resistance against insect attack, pathogen infection, and high temperature stress (Hu et al., 2013; Yan et al., 2013). Pepper CaLOX1 was involve in defence and cell-death responses against pathogens (Hwang and Hwang, 2010), and transgenic Arabidopsis plants overexpressing CaLOX1 resulted in enhanced tolerance to osmotic, drought, and high salinity stress (Lim et al., 2015). Another gene, CaLOX2, was involved in the JA biosynthesis and resistance of cultivated pepper to Western flower thrips (Frankliniella occidentalis) (Sarde et al., 2019). In addition, overexpression of persimmon DKLOX3 in Arabidopsis promoted resistance to drought, high salinity and osmotic stress via regulating reactive oxygen species accumulation and stress responsive genes expression (Hou et al., 2015). Arabidopsis plants overexpressing oriental melon $C m L O X 13$ also exhibited enhanced resistance to drought stress via regulating abscisic acid (ABA) accumulation and stomatal closure (Xing et al., 2019).

In our previous study, a total of $23 L O X$ genes were identified in cucumber genome and their tissue expression patterns were examined (Liu et al., 2011). Subsequently, the expression profiling of the $L O X$ genes during fruit development, various abiotic stress and hormonal treatments were also determined (Yang et al., 2012). And a previous report showed that the yellow-green leaf (ygli) mutant was due to mutations in four tandem cucumber 13-LOX genes (Ding et al., 2019). At the different storage temperatures, the expression of CsLOX genes might led to the differences in the contents of six-carbon (C6) and nine-carbon (C9) aldehydes (Yang et al., 2020). However, the detailed functional analysis for cucumber $L O X$ genes is still limited. In this work, a cucumber $L O X$ gene (CsLOXI) was isolated and it was overexpressed in transgenic Arabidopsis plants, to investigate its potential function. Our findings indicated that $C s L O X 1$ plays important roles in the growth and development of cucumber.

\section{Materials and Methods}

\section{Plant materials and growth conditions}

Cucumber (Cucumis sativus L. cv 'Chinese long' 9930 inbred line) plants were grown in a climate chamber at a night temperature of $18{ }^{\circ} \mathrm{C}$ and day temperature of $24^{\circ} \mathrm{C}$ under long-day conditions $(16 \mathrm{~h}$ of light $/ 8 \mathrm{~h}$ of dark). Flower samples of 20 main-stem nodes stages were collected, immediately frozen in liquid nitrogen, and stored at $-80{ }^{\circ} \mathrm{C}$ until use.

Wild-type (WT, Col ecotype) and transgenic Arabidopsisseeds were placed $4{ }^{\circ} \mathrm{C}$ under dark conditions for dormancy breaking. And then the seeds were germinated and planted in a growth room at $24 / 18{ }^{\circ} \mathrm{C}$ (day/night) under long-day conditions ( $16 \mathrm{~h}$ of light/ $8 \mathrm{~h}$ of dark). 
$R N A$ extraction and $c D N A$ synthesis

Total RNA was extracted using Trizol reagent (Tiangen Biotech, Beijing, China) according to the manufacturer's instruction. Subsequently, the integrity of the RNA samples was checked on a $1.0 \%$ agarose gel. After concentration analysis using Nanodrop 2000 (Thermo Fisher Scientific, USA), about $3 \mu \mathrm{g}$ RNA was reverse transcribed as cDNA using the M-MLV reverse transcriptase (Invitrogen, USA) based on the manufacturer's protocol.

\section{Cloning and sequence analysis of the CsLOX1 gene}

For cloning the $C s L O X 1$ gene, cDNA from cucumber flower samples was prepared as the template. The specific primers CsLOX1-1F (5'-aaaaCTGCAGATGTTTGGAATTGGGAAGAACAT-3') and CsLOX1$1 \mathrm{R}\left(5^{\prime}\right.$-aaaaTCTAGATTAGATAGAAATACTATTAGGAAT-3') were designed based on the open reading frame (ORF) sequence of CsLOX1 (gene ID: Csa006735) in previous reports (Liu et al., 2011; Yang et al., 2012). The CsLOX1 ORF was amplified with semiquantitative reverse-transcription polymerase chain reaction (RT-PCR). The PCR procedure was carried out as follows: 1 cycle at $94^{\circ} \mathrm{C}$ for $5 \mathrm{~min}$, followed by 30 cycles of $1 \mathrm{~min}$ at $94^{\circ} \mathrm{C}, 1 \mathrm{~min}$ at $59^{\circ} \mathrm{C}$, and $3 \mathrm{~min}$ at $72{ }^{\circ} \mathrm{C}$, and then a final extension at $72{ }^{\circ} \mathrm{C}$ for $10 \mathrm{~min}$. Then, the PCR products were inserted into the pMD18-T vector (Takara, Japan) and then sequenced (Tsingke, Beijing, China).

\section{Sequence analysis of the CsLOX1 gene and the deduced CsLOX1 protein}

The exon-intron of CSLOX1 was analyzed by Gene Structure Display Server 2.0 (GSDS, http://gsds.cbi.pku.edu.cn/) comparing the sequences of coding sequence (CDS) and genome DNA (gDNA). The theoretical isoelectric point ( $\mathrm{pI}$ ) and molecular weight $(\mathrm{MW})$ were calculated with protparam (https://web.expasy.org/protparam/). Subcellular localization of CsLOX1 was predicted by using online tools Plant-mPLoc (http://www.csbio.sjtu.edu.cn/cgi-bin/PlantmPLoc.cgi) and ProtComp Version 9.0 (http://linux 1.softberry.com/berry.phtml?topic=protcomppl\&group=programs\&subgroup=proloc). $\quad$ For promoter region analysis, the $2.0-\mathrm{kb}$ upstream sequences of the start codon of the CsLOX1 gene was submitted into the PlantCARE online tool (http://bioinformatics.psb.ugent.be/webtools/plantcare/html/), and the putative development-, stress-, and hormone-related cis-elements were analyzed.

\section{Sequence alignment and phylogenetic analysis}

The multiple sequence alignment of the deduced amino acid sequences of CsLOX1 and other plant LOX proteins were carried out using the Clustal Omega program (https://www.ebi.ac.uk/Tools/msa/clustalo/), and then the align results were displayed with the GeneDoc software as described previously (Zhou et al., 2019b). The MEGA 7.0 software was employed to create a neighbour-joining $(\mathrm{NJ})$ phylogenetic tree using the same align results with the following parameters: 1000 bootstrap replicates, poisson model, pairwise deletion.

\section{Vector construction, Arabidopsis transformation and morphological observation}

The sequencing verified pMD18-T vector carrying CsLOX1 ORF was digested with Pst I/Xba I, and then the digested fragment was inserted into the $\mathrm{P}$ st $\mathrm{I} / \mathrm{Xba}$ I restriction sites downstream of the double $35 \mathrm{~S}$ promoter of the $\mathrm{pHB}$ vector (Zhou et al., 2017). The overexpression construct was named as 35S::35S::CsLOX1, and Arabidopsis transformation was conducted by Agrobacterium tumefaciens-mediated floral dip method as described previously (Zhou et al., 2019b). The transgenic plants were checked with RTPCR using the following CsLOXI-specific primers: 5'-AGGACCTCACTCCACCTTTG-3' and 5'AACCGTAAGACCATCTAAACCAT-3'. The AtTubulint 4 gene is a reference gene, and its primers sequences are as follows: 5'-GCGAACAGTTCACAGCTATGTTCA-3' and 5'GAGGGAGCCATTGACAACATCTT-3'. The reaction conditions were performed as described above. The transgenic plants exhibit increased $C s L O X 1$ expression were used for morphological observation. 


\section{Results and Discussion}

Isolation and sequence analysis of CSLOX1 in cucumber

Using cucumber flower cDNA as the template, a 2637-bp PCR fragment was amplified with the specific CsLOX1-1F and CsLOX1-1R primers. For investigating the chromosomal localization of the CsLOX1 gene, we carried out Blastn search against the Cucumber (Chinese Long) v3 Genome (http://cucurbitgenomics.org/organism/20). The result indicated that the Locus ID of CSLOXI was CsaV3_2G006380 and it was located on chromosome 2. In addition, the CsLOX1 gene encoded a 878 aminoacid residue protein, with a pI value of 6.04 , and a MW value of $99.78 \mathrm{kDa}$. The GSDS analysis showed that CsLOXI harbored 9 exons and 8 introns (Figure 1A). The SMART analysis showed that CsLOX1 possessed two conserved domains, PLAT (Polycystin-1, Lipoxygenase, Alpha-Toxin) or LH2 (Lipoxygenase homolog) (SMART Accession: SM000308) and lipoxygenase domain (Pfam Accession: PF00305), which were located between 40-181 and 192-861 amino acids, respectively (Figure 1B). SOPMA analysis showed that the secondary structure of CsLOX1 was composed by $39.07 \%$ alpha helix, $13.10 \%$ extended strand, $5.13 \%$ beta turn, and $42.71 \%$ random coil (Figure 1C). Plant LOX proteins are found to have various subcellular localizations, such as chloroplast, cytoplasm and vacuole. And 9-LOXs were usually found to localized in cytoplasm, while many 13-LOXs were localized in chloroplast (Upadhyay and Mattoo, 2018; Zhu et al., 2018). In this work, both of Plant-mPLoc and ProtComp subcellular prediction analysis showed that CsLOX1 was located in cytoplasm. These results indicated that CsLOX1 is a typical cytoplasmic lipoxygenase protein.
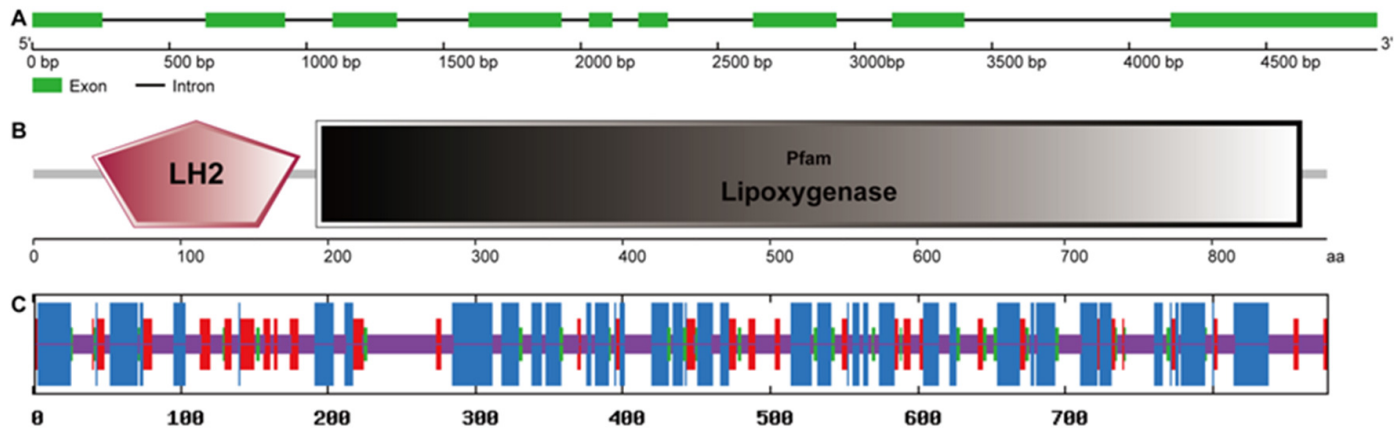

Figure 1. Sequence analysis of $C S L O X 1$ and its deduced amino acid sequences

(A) Exon-intron structure of the CsLOX1 gene. (B) SMART analysis of the conserved domains of CsLOX1. (C) SOPMA analysis of the secondary structure of CsLOX1. The blue, red, green, and purple regions indicate of alpha helix, extended strand, beta turn, and random coil, respectively.

\section{Characterization and phylogenetic analysis of CsLOXI with other LOX proteins}

To characterize the features of CsLOX1, a multiple alignment analysis was performed based on the amino acid sequences of the putative CsLOX1 and other plant LOX proteins, including olive (Olea europaea) Oep2LOX1 (Padilla et al., 2012), maize (Zea mays) ZmLOX12 (Christensen et al., 2014), rice (Oryza sativa) OsLOX2 (Huang et al., 2014), persimmon (Diospyros kaki) DkLOX3 and DkLOX4 (Hou et al., 2015; Meng et al., 2016), agarwood (Aquilaria sinensis) AsLOX1 (Liao et al., 2015), finger millet (Eleusine coracana) EcLOX (Kotapati et al., 2016), melon (Cucumis melo) CmLOX09 (Ju et al., 2018) and CmLOX13 (Xing et al., 2019; Cao et al., 2016), pepper (Capsicum annuum) CaLOX1 (Hwang and Hwang, 2010; Lim et al., 2015) and CaLOX2 (Sarde et al., 2019). The alignment analysis results revealed that CsLOX1 shared relatively high identities to these LOX proteins, ranged from $41.80 \%$ (CmLOX13) to $62.27 \%$ (CaLOX1) (Figure 2). All of these LOX proteins shared the conserved regions such as LH2/PLAT domain, lipoxygenase domain, and highly conserved C-terminal motif. In addition, all of these LOX proteins contained the representative 38 amino acids motif [His-(X)4-His-(X)4-His-(X)17-His-(X)8-His] for enzyme stability and activity, which was provide binding sites for non-haeme iron-containing dioxygenases (Shaban et al., 2018). In addition, three His 
$(\mathrm{H})$, one Asn $(\mathrm{N})$ and one Ile $(\mathrm{I})$ residues involved in the binding of the iron atom in the active site, were also present in CsLOX1 and LOX proteins from other plants (Padilla et al., 2012; Christensen et al., 2014; Kotapati et al., 2016). Previous reports showed that Val (V) residue in the conserved motif may determine 9LOX regio-specific activity, while Phe (F) residue indicative of LOX enzymes with 13-LOX activity ( Liavonchanka and Feussner, 2006; Kotapati et al., 2016). However, CsLOX1 have a His (H) at position 597 (Figure 2), which is different from the characterization of 9-LOX, suggesting that CsLOX1 may have special roles in cucumber. And previous studies showed that several 9-LOX proteins also had no Val residue at this positions (Christensen et al., 2014; Li et al., 2012). Similar to other LOX proteins, the conserved Ala (A) residue at position 582 was also detected in CsLOX1, which is required for substrate orientation and the Sstereo-specificity ( Padilla et al., 2012; Kotapati et al., 2015). And the Arg (R) residue at position 747 can function in interacting with the carboxyl group of the fatty acid and required for the inverse substrate orientation in plant LOXs (Kotapati et al., 2015). Moreover, CsLOX1 harbores two conservative motifs which have been shown to be essential for substrate (GWSTDEEFAREMLAG) and oxygen binding (ASALHAAVNFGQY) (Figure 2).

To further study the phylogenetic relationships between CsLOX1 and other plant LOX proteins, a phylogenetic tree was created by aligning LOX protein sequences from various plant species. Our phylogenetic analysis showed that these LOXs can be divided into two groups: 9-LOX and 13-LOX, and CsLOX1 was fall into the 9-LOX group with other dicot 9-LOXs (Figure 3), demonstrating that CsLOX1 is a 9-LOX.

\section{Cis-element analysis of CsLOXI}

In consideration of the cis-acting regulatory elements in the promoter region is important for understanding the expression patterns of genes (Zhou et al., 2020), we investigated the distribution of putative development-, stress-, and hormone-related cis-elements in the 2.0-kb upstream sequences of the start codon of the CsLOXI gene using PlantCARE. As shown in Figure 4, one ABA responsiveness element (ABRE) and three ethylene-responsive elements (ERE) were present in the promoter region of CsLOXI, which were correlated with the expression of $C s L O X 1$ in response to $\mathrm{ABA}$ and ethylene. In a previous study, $C s L O X 1$ was observably induced by exogenous $\mathrm{ABA}$ and 1-aminocyclopropane-l-carboxylic acid (ACC; precursor of ethylene), and its mRNA accumulations were peaked at $12 \mathrm{~h}$ after treatment (almost 40 -fold) by ABA and $6 \mathrm{~h}$ after treatment (almost 17-fold) by ACC (Yang et al., 2012). In addition, two types of cis-elements in stress responsive, ARE and TC-rich repeats, which were take part in anaerobic induction, defense and stress responsiveness, respectively, were found in promoter region of CsLOXI (Figure 4). And CsLOXI was also significantly induced by $\mathrm{NaCl}$ and $\mathrm{KCl}$ treatments (Yang et al., 2012). When plants were subjected to abiotic stresses, endogenous ABA levels were increased to help plants adapt to the stress conditions (Zhou et al., 2020; Vishwakarma et al., 2017). And some $L O X$ genes can enhanced abiotic stresses resistance by modulating the ABA-responsive genes and promoting the endogenous ABA levels, such as CaLOX1 (Lim et al., 2015), DkLOX3 (Hou et al., 2015), and CmLOX13 (Xing et al., 2019). Therefore, CsLOX1 may also play a role in resistance to one or more stresses via ABA-dependent pathway. Previous reports showed that plant $L O X$ genes can be regulated by circadian rhythms (Nemchenko et al., 2006; Zhu et al., 2018). In this study, CsLOXI promoter also harbored one circadian cis-element related to circadian control, suggesting that CsLOXI might be involved in circadian rhythms regulation in cucumber. 

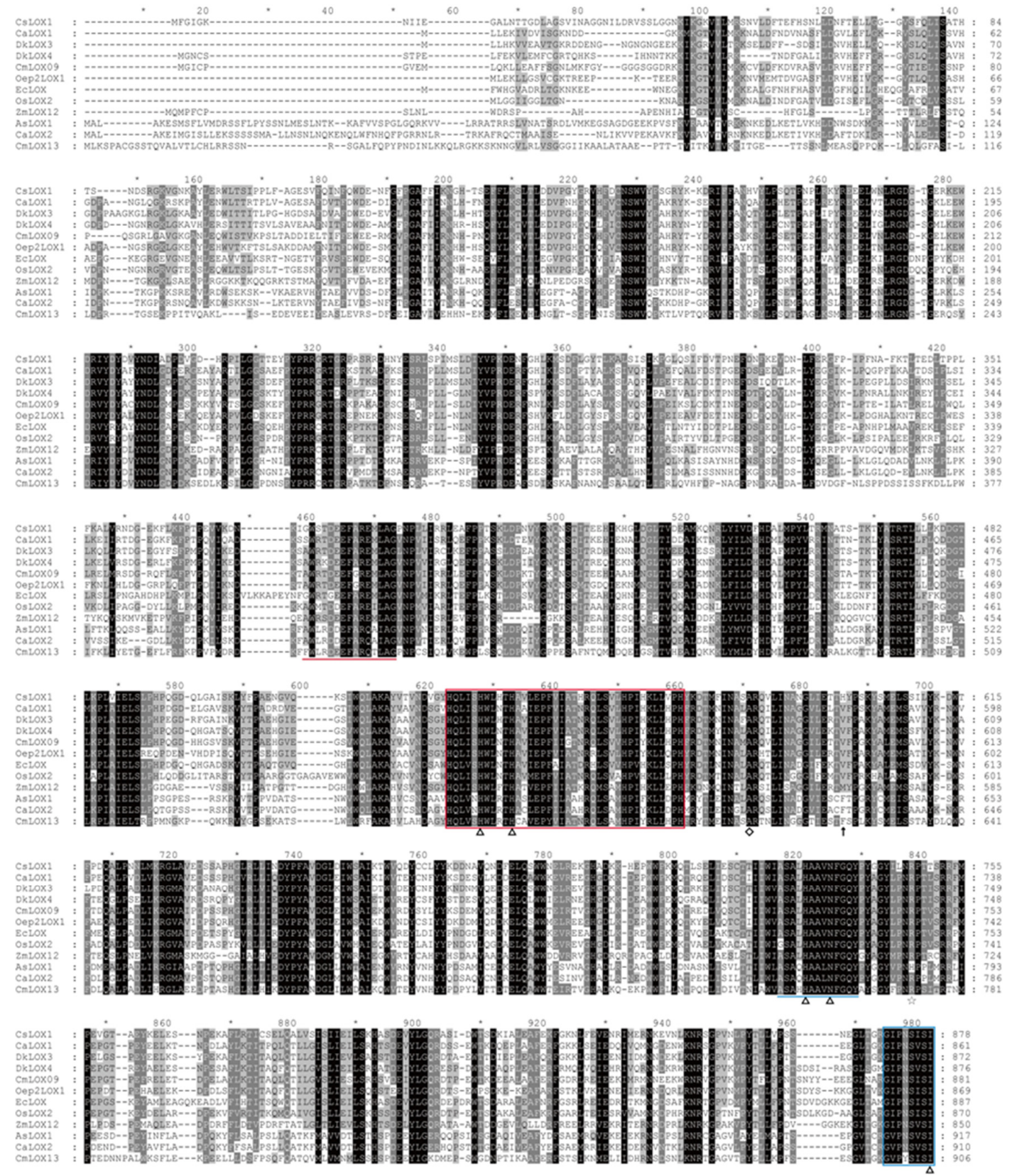

Figure 2. Alignment of the deduced amino acid sequences of CsLOX1 and LOX proteins from other plants

These sequences were aligned using Clustal Omega and visualized in the GeneDoc software. The representative 38 amino acids motif [His-(X)4-His-(X)4-His-(X)17-His-(X)8-His] and highly conserved C-terminal motif are boxed with red and blue, respectivley. Two conserved motifs required for substrate (GWSTDEEFAREMLAG) and oxygen binding (ASALHAAVNFGQY) were underlined with red and blue, respectivley. The five conserved residues (His539, His544, His730, Asn734, and Ile878) essential for the binding of the iron atom in the active site of the LOXs enzymes are indicated by triangles. The Val $(\mathrm{V})$ or Phe $(\mathrm{F})$ residues indicative of LOX enzymes with 9-LOX or 13-LOX activities are marked by arrow. The Ala582 that required for substrate orientation and the S-stereo-specificity is marked by rhombus, and the Arg747 determinant for the inverse substrate orientation in plant LOXs is marked by pentacle. The details of CsLOX1 and other plant LOX proteins were listed in Table S1. 


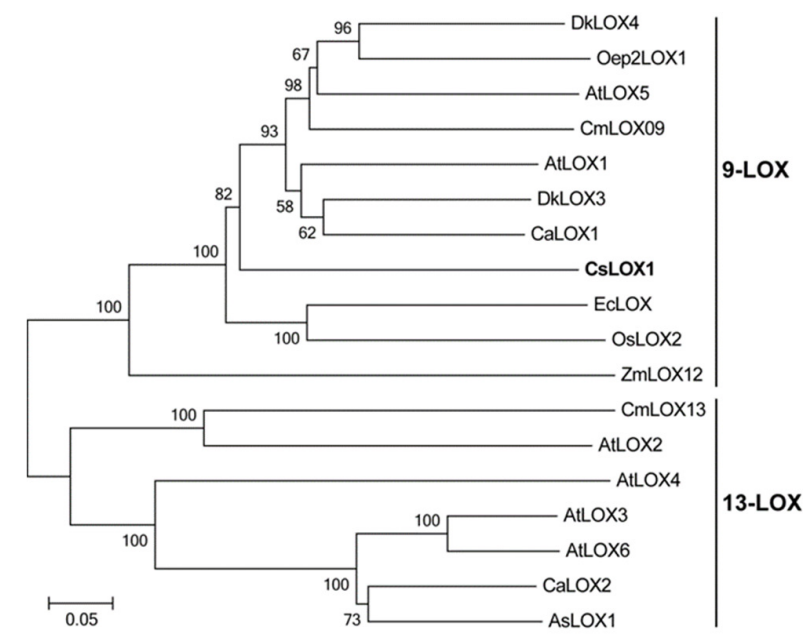

Figure 3. Phylogenetic relationships of CsLOX1 and other plant LOX proteins. LOX proteins from various plants were aligned with Clustal Omega, and the align results were employed to generate the phylogenetic tree with MEGA 7.0 using NJ method

The bootstrap test was set as 1000 replicates. The details of CsLOX1 and other plant LOX proteins were listed in Table S1. CsLOX1 is bolded.

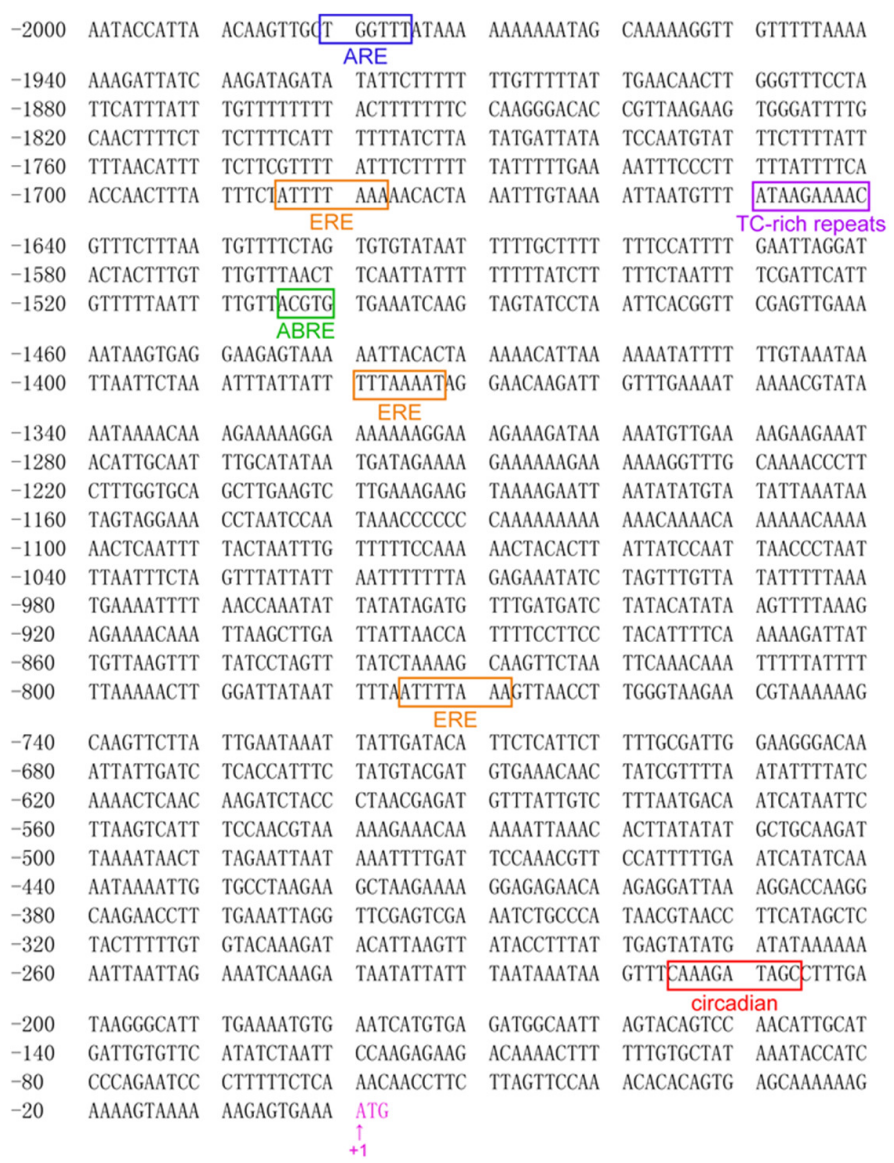

Figure 4. Cis-acting regulatory element analysis of CsLOX1

Putative cis-elements in the promoter region of $C s L O X 1$ was boxed and colored. The "A" of the start codon "ATG" of $C s L O X 1$ was designated as +1 position. 


\section{Overexpression of CSLOXI causes male sterility in transgenic Arabidopsis plants}

Previous studies revealed $C S L O X 1$ have a constitutive expression pattern, but its expression was preferentially detected in ovaries and early developing fruits, implying its particular role in fruit development immediately after anthesis (Liu et al., 2011; Yang et al., 2012). To further uncover its role in cucumber, the overexpression vector 35S::35S::CsLOX1 was constructed by inserting the ORF of CsLOX1 into the pHB vector, under the control of double CaMV $35 \mathrm{~S}$ promoter (Figure 5A). The overexpression construct 35S::35S::CsLOX1 was transformed into Arabidopsis. Three transgenic lines (OE1, OE2, and OE3) were chosen for further study, and RT-PCR showed that $C s L O X 1$ was highly expressed in these transgenic plants while no expression was detected in WT plants (Figure 5B).

To study how the overexpression of $C s L O X 1$ in transgenic plants, we investigate the phenotypes of the transgenic Arabidopsis plants. At stage 13, the transgenic plants exhibited green anthers, and the filaments were much slenderer compared to the WT plants (Figure 6A-C), suggesting that the stamen development was impaired in transgenic plants. And all seeds of these transgenic plants are fully sterile. However, it has no impact on the development of other floral organs, such as pistils, sepals, and petals. Hence, the complete male sterility of transgenic Arabidopsis plants was induced by the overexpression of CsLOX1. In a previous study, Arabidopsis single lox 3 and lox 4 mutants were found to be fertile, while lox 3 lox 4 double mutants were male sterile (Caldelari et al., 2011). In addition, it was observed that the transgenic Arabidopsis plants displayed obviously increased floral shoots compared to the WT plants (Figure 6D-E), and these phenotypes were also observed in lox3 lox4 plants (Caldelari et al., 2011). Therefore, overexpression of CsLOX1 may impair the endogenous JA production and then inhibit the expression of Arabidopsis $L O X$ genes. Therefore, the role of CsLOXI may affect plant growth and development, such as male fertility, shoot growth and fruit development.

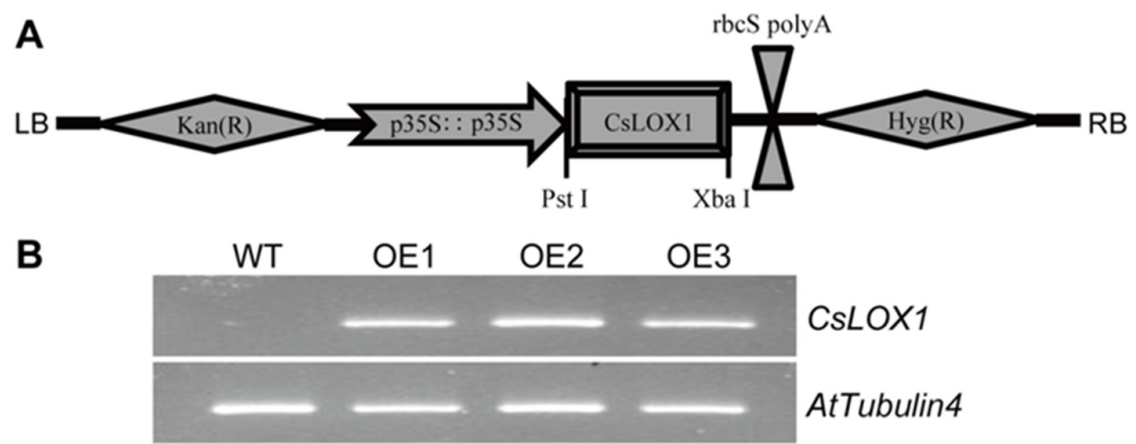

Figure 5. Analysis of transgenic Arabidopsis lines overexpressing the CsLOX1 gene (A) Schematic diagram of the T-DNA region of 35S::35S::CsLOX1 overexpression construct. (B) RT-PCR analysis of expression of CSLOX1 in WT and transgenic Arabidopsis plants. WT, wild-type Arabidopsis plants. OE1-3, 35S::35S::CsLOX1 transgenic Arabidopsis lines. AtTubulin4 gene was used as the internal control. 

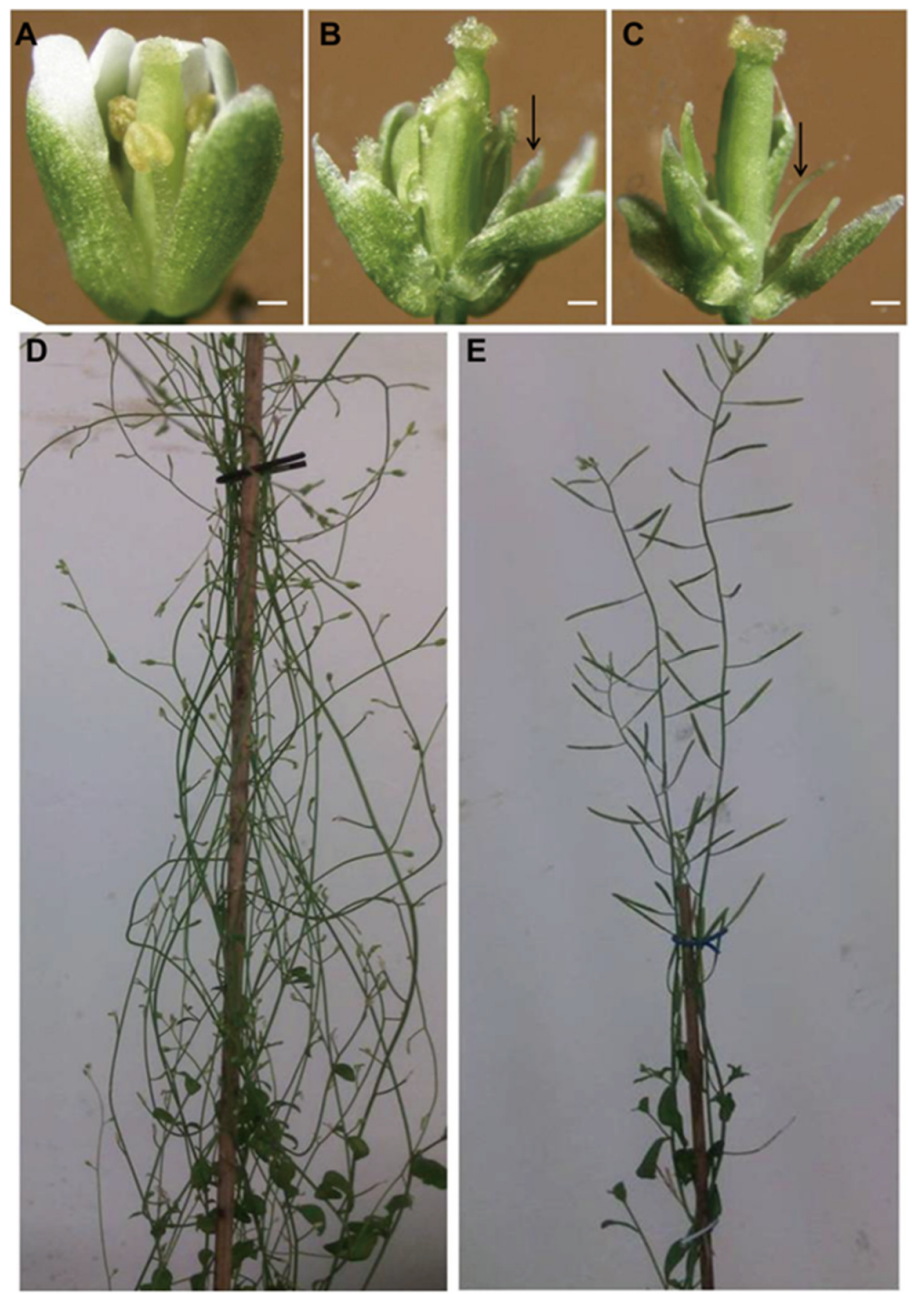

Figure 6. Comparison of the phenotypes of 35S::35S::CsLOX1 transgenic Arabidopsis plants

(A, E) WT Arabidopsis plants and (B-D) 35S::35S::CsLOX1 plants. (A) The flower buds of WT plant at stage 12. (B) Green anthers (arrow) at stage 13. (C) Filaments become slenderer than those of WT plants (arrow). (D) One 35S::35S::CsLOX1 transgenic plant at stage 17. (E) One WT plant at stage 17.

\section{Conclusions}

In this work, a cucumber $L O X$ gene $(C s L O X I)$ was isolated and its features were characterized by bioinformatics approaches. This gene encodes a typical cytoplasmic 9-LOX, and possesses the typical LH2/PLAT and lipoxygenase domains, as well as essential conserved residues. And transgenic analysis in Arabidopsis was carried out to investigate the possible functions of CsLOX1. Overexpression of CsLOX1 in Arabidopsis induced a phenotype of male sterility that resulted from the failure of stamen development. In addition, the transgenic plants displayed obviously increased floral shoots compared to the WT plants. Our findings provide a basis for revealing of the biological roles of $C s L O X 1$ in cucumber. 


\section{Authors' Contributions}

Conceptualization: SL; Data curation: YZ and SL; Formal analysis: YZ, MX, WL and SL; Funding acquisition: SL; Investigation: YZ, MX, WL, LC, YY and SL; Methodology: YZ, MX, WL, LC, YY and SL; Resources: YZ and SL; Software: YZ, WL and SL; Visualization; Writing - original draft: YZ and SL; Writing - review and editing: YZ, YY and SL. All authors read and approved the final manuscript.

\section{Acknowledgements}

This work was funded by the National Natural Science Foundation of China (31060262 and 31660578).

\section{Conflict of Interests}

The authors declare that there are no conflicts of interest related to this article.

\section{References}

Acosta IF, Laparra H, Romero SP, Schmelz E, Hamberg M, Mottinger JP . . Dellaporta SL (2009). Tasselseed1 is a lipoxygenase affecting jasmonic acid signaling in sex determination of maize. Science 323(5911):262-265. https://doi.org/10.1126/science.1164645

Bannenberg G, Martinez M, Hamberg M, Castresana C (2009). Diversity of the enzymatic activity in the lipoxygenase gene family of Arabidopsis thaliana. Lipids 44(2):85-95. https://doi.org/10.1007/s11745-008-3245-7

Brodhun F, Feussner I (2011). Oxylipins in fungi. The FEBS Journal 278(7):1047-1063. https://doi.org/10.1111/j.17424658.2011.08027.x

Caldelari D, Wang G, Farmer EE, Dong X (2011). Arabidopsis lox3 lox4 double mutants are male sterile and defective in global proliferative arrest. Plant Molecular Biology 75(1-2):25-33. https://doi.org/10.1007/s11103-010-9701-9

Cao S, Chen H, Zhang C, Tang Y, Liu J, Qi H (2016). Heterologous expression and biochemical characterization of two lipoxygenases in oriental melon, Cucumis melo var. makuwa Makino. PLoS One 11(4):e0153801. https://doi.org/10.1371/journal.pone.0153801

Christensen SA, Nemchenko A, Park YS, Borrego E, Huang PC, Schmelz EA . . Kolomiets MV (2014). The novel monocot-specific 9-lipoxygenase $\mathrm{ZmLOX} 12$ is required to mount an effective jasmonate-mediated defense against Fusarium verticillioides in maize. Molecular Plant-Microbe Interactions 27(11):1263-1276. https://doi.org/10.1094/MPMI-06-13-0184-R

Ding Y, Yang W, Su C, Ma H, Pan Y, Zhang X, Li J (2019). Tandem 13-lipoxygenase genes in a cluster confers yellowgreen leaf in cucumber. International Journal of Molecular Sciences 20(12):3102. https://doi.org/10.3390/ijms20123102

Feussner I, Wasternack C (2002). The lipoxygenase pathway. Annual Review of Plant Biology 53:275-297. https://doi.org/10.1146/annurev.arplant.53.100301.135248

Guo S, Song Z, Ma R, Yang Y, Yu M (2017). Genome-wide identification and expression analysis of the lipoxygenase gene family during peach fruit ripening under different postharvest treatments. Acta Physiologiae Plantarum 39(5):111. https://doi.org/10.1007/s11738-017-2409-6

Hayashi S, Gresshoff PM, Kinkema M (2008). Molecular analysis of lipoxygenases associated with nodule development in soybean. Molecular Plant-Microbe Interactions 21(6):843-853. https://doi.org/10.1094/MPMI-21-6-0843

Hou Y, Meng K, Han Y, Ban Q, Wang B, Suo J . . Rao J (2015). The persimmon 9-lipoxygenase gene DkLOX3 plays positive roles in both promoting senescence and enhancing tolerance to abiotic stress. Frontiers in Plant Science 6:1073. https://doi.org/10.3389/fpls.2015.01073 
Hu T, Zeng H, Hu Z, Qv X, Chen G (2013). Overexpression of the tomato 13-lipoxygenase gene TomloxD increases generation of endogenous jasmonic acid and resistance to cladosporium fulvum and high temperature. Plant Molecular Biology Reporter 31(5):1141-1149. https://doi.org/10.1007/s11105-013-0581-4

Huang J, Cai M, Long Q, Liu L, Lin Q, Jiang L ... Wan J (2014). OsLOX2, a rice type I lipoxygenase, confers opposite effects on seed germination and longevity. Transgenic Research 23(4):643-655. https://doi.org/10.1007/s11248014-9803-2

Hwang IS, Hwang BK (2010). The pepper 9-lipoxygenase gene CaLOX1 functions in defense and cell death responses to microbial pathogens. Plant Physiology 152(2):948-967. https://doi.org/10.1104/pp.109.147827

Ju LJ, Zhang C, Liao JJ, Li YP, Qi HY (2018). An oriental melon 9-lipoxygenase gene CmLOX09 response to stresses, hormones, and signal substances. Journal of Zhejiang University-Science B 19(8):596-609. https://doi.org/10.1631/jzus.b1700388

Kolomiets MV, Hannapel DJ, Chen H, Tymeson M, Gladon RJ (2001). Lipoxygenase is involved in the control of potato tuber development. The Plant Cell 13(3):613-626. https://doi.org/10.1105/tpc.13.3.613

Kotapati KV, Palaka BK, Anangi R, Kedam T, Ampasala DR (2015). Cloning and characterization of lipoxygenase gene from germinating seedlings of green gram (Vigna radiata L.). Indian Journal of Plant Physiology 20(4):345-352.

Kotapati KV, Palaka BK, Kandukuri A, Pamuru RR, Lebaka VR, Ampasala DR (2016). Molecular cloning, characterization and three-dimensional structure prediction of lipoxygenase from finger millet [Eleusine coracana (L.) Gaertn.] germinating seedlings. Journal of Plant Biochemistry and Biotechnology 25(2):155-167.

Li M, Li L, Dunwell JM, Qiao X, Liu X, Zhang S (2014). Characterization of the lipoxygenase (LOX) gene family in the Chinese white pear (Pyrus bretschneideri) and comparison with other members of the Rosaceae. BMC Genomics 15(1):444. https://doi.org/10.1186/1471-2164-15-444

Li ST, Zhang M, Fu C-h, Xie S, Zhang Y, Yu LJ (2012). Molecular cloning and characterization of two 9-lipoxygenase genes from Taxus chinensis. Plant Molecular Biology Reporter 30(6):1283-1290. https://doi.org/10.1007/s11105-012-0439-1

Liao YC, Xu YH, Zhang Z, Wei JH (2015). Molecular cloning and sequence analysis of lipoxygenase $(A s L O X 1)$ gene in Aquilaria sinensis (Lour.) Gilg and its expression responding to MeJA and crush wounding treatment. Plant Gene 2:10-16.

Liavonchanka A, Feussner I (2006). Lipoxygenases: occurrence, functions and catalysis. Journal of Plant Physiology 163(3):348-357. https://doi.org/10.1016/j.jplph.2005.11.006

Lim CW, Han SW, Hwang IS, Kim DS, Hwang BK, Lee SC (2015). The pepper lipoxygenase CaLOX1 plays a role in osmotic, drought and high salinity stress response. Plant and Cell Physiology 56(5):930-942. https://doi.org/10.1093/pcp/pcv020

Liu S, Han B (2010). Differential expression pattern of an acidic 9/13-lipoxygenase in flower opening and senescence and in leaf response to phloem feeders in the tea plant. BMC Plant Biology https://doi.org/10:228. 10.1186/14712229-10-228

Liu SQ, Liu XH, Jiang LW (2011). Genome-wide identification, phylogeny and expression analysis of the lipoxygenase gene family in cucumber. Genetics and Molecular Research 10(4):2613-2636. https://doi/org/10.4238/2011.october.25.9

Meng K, Hou Y, Huber DJ, Dong X, Ban Q, Chang X... Rao J (2016). Molecular cloning, structural characterization, and ripening-related expression of lipoxygenase genes from three persimmon cultivars differing in postharvest ripening rate. Plant Molecular Biology Reporter 34(4):854-868. https://doi.org/10.1007/s11105-015-0971-X

Nemchenko A, Kunze S, Feussner I, Kolomiets M (2006). Duplicate maize 13-lipoxygenase genes are differentially regulated by circadian rhythm, cold stress, wounding, pathogen infection, and hormonal treatments. Journal of Experimental Botany 57(14):3767-3779. https://doi.org/10.1093/jxb/erl137

Padilla MN, Hernández ML, Sanz C, Martínez-Rivas JM (2012). Molecular cloning, functional characterization and transcriptional regulation of a 9-lipoxygenase gene from olive. Phytochemistry 74:58-68. https://doi.org/10.1016/j.phytochem.2011.11.006

Porta H, Rocha-Sosa M (2001). Lipoxygenase in bacteria: a horizontal transfer event? Microbiology 147(Pt 12):31993200. https://doi.org/10.1099/00221287-147-12-3199

Sarde SJ, Bouwmeester K, Venegas-Molina J, David A, Boland W, Dicke M (2019). Involvement of sweet pepper CaLOX2 in jasmonate-dependent induced defence against Western flower thrips. Journal of Integrative Plant Biology 61(10):1085-1098. https://doi.org/10.1111/jipb.12742 
Sarde SJ, Kumar A, Remme RN, Dicke M (2018). Genome-wide identification, classification and expression of lipoxygenase gene family in pepper. Plant Molecular Biology 98(4-5):375-387. https://doi.org/10.1007/s11103018-0785-y

Shaban M, Ahmed MM, Sun H, Ullah A, Zhu L (2018). Genome-wide identification of lipoxygenase gene family in cotton and functional characterization in response to abiotic stresses. BMC Genomics 19(1):599. https://doi.org/10.1186/s12864-018-4985-2

Springer A, Kang C, Rustgi S, von Wettstein D, Reinbothe C, Pollmann S, Reinbothe S (2016). Programmed chloroplast destruction during leaf senescence involves 13-lipoxygenase (13-LOX). Proceedings of The National Academy of Sciences of The United States of America 113(12):3383-3388. https://doi.org/10.1073/pnas.1525747113

Umate P (2011). Genome-wide analysis of lipoxygenase gene family in Arabidopsis and rice. Plant Signaling Behavior 6(3):335-338. https://doi.org/10.4161/psb.6.3.13546

Upadhyay RK, Mattoo AK (2018). Genome-wide identification of tomato (Solanum lycopersicum L.) lipoxygenases coupled with expression profiles during plant development and in response to methyl-jasmonate and wounding. Journal of Plant Physiology 231:318-328. https://doi.org/10.1016/j.jplph.2018.10.001

Vishwakarma K, Upadhyay N, Kumar N, Yadav G, Singh J, Mishra RK ... Sharma S (2017). Abscisic acid signaling and abiotic stress tolerance in plants: a review on current knowledge and future prospects. Frontiers in Plant Science 8:161. https://doi.org/10.3389/fpls.2017.00161

Viswanath KK, Varakumar P, Pamuru RR, Basha SJ, Mehta S, Rao AD (2020). Plant lipoxygenases and their role in plant physiology. Journal of Plant Biology 63(2):83-95.

Vogt J, Schiller D, Ulrich D, Schwab W, Dunemann F (2013). Identification of lipoxygenase (LOX) genes putatively involved in fruit flavour formation in apple (Malus $\times$ domestica). Tree Genetics \& Genomes 9(6):1493-1511. https://doi.org/10.1007/s11295-013-0653-5

Wang J, Hu T, Wang W, Hu H, Wei Q, Wei X, Bao C (2019). Bioinformatics analysis of the lipoxygenase gene family in radish (Raphanus sativus) and functional characterization in response to abiotic and biotic stresses. International Journal of Molecular Sciences 20(23):6095. https://doi.org/10.3390/ijms20236095

Wang R, Shen W, Liu L, Jiang L, Liu Y, Su N, Wan J (2008). A novel lipoxygenase gene from developing rice seeds confers dual position specificity and responds to wounding and insect attack. Plant Molecular Biology 66(4):401-414. https://doi.org/10.1007/s11103-007-9278-0

Wasternack C, Feussner I (2018). The oxylipin pathways: biochemistry and function. Annual Review of Plant Biology 69:363-386. https://doi.org/10.1146/annurev-arplant-042817-040440

Xing Q, Zhang X, Li Y, Shao Q, Cao S, Wang F, Qi H (2019). The lipoxygenase CmLOX13 from oriental melon enhanced severe drought tolerance via regulating ABA accumulation and stomatal closure in Arabidopsis. Environmental and Experimental Botany 167:103815.

Yamamoto S (1992). Mammalian lipoxygenases: molecular structures and functions. Biochimica et Biophysica Acta 1128(2-3):117-131. https://doi.org/10.1016/0005-2760(92)90297-9

Yan L, Zhai Q, Wei J, Li S, Wang B, Huang T ... Li C (2013). Role of tomato lipoxygenase D in wound-induced jasmonate biosynthesis and plant immunity to insect herbivores. PLoS Genetics 9(12):e1003964. https://doi.org/10.1371/journal.pgen.1003964

Yang XY, Jiang WJ, Yu HJ (2012). The expression profiling of the lipoxygenase (LOX) family genes during fruit development, abiotic stress and hormonal treatments in cucumber (Cucumis sativus L.). International Journal of Molecular Sciences 13(2):2481-2500. https://doi.org/10.3390/ijms13022481

Yang Y, Zhang T, Wang X, Wu M, Li X, Guo X, Fang Y, Chen S (2020). Relationship between CsLOX gene expression and C6 and C9 aldehydes during cucumber fruit storage. Postharvest Biology and Technology 161:111085.

Zhang C, Cao S, Jin Y, Ju L, Chen Q, Xing Q, Qi H (2017). Melon 13-lipoxygenase CmLOX18 may be involved in C6 volatiles biosynthesis in fruit. Scientific Reports 7(1):2816. https://doi.org/10.1038/s41598-017-02559-6

Zhou Y, Ge L, Li G, He P, Yang Y, Liu S (2020). In silico identification and expression analysis of rare cold inducible 2 (RCI2) gene family in cucumber. Journal of Plant Biochemistry and Biotechnology 29:56-66. https://doi.org/10.1007\%2Fs13562-019-00510-6

Zhou Y, Guang Y, Li J, Wang F, Ahammed GJ, Yang Y (2019a). The CYP74 gene family in watermelon: Genome-wide identification and expression profiling under hormonal stress and root-knot nematode infection. Agronomy-Basel 9(12):872. https://doi.org/10.3390/agronomy9120872

Zhou Y, Hu L, Jiang L, Liu H, Liu S (2017). Molecular cloning and characterization of an ASR gene from Cucumis sativus. Plant Cell Tissue and Organ Culture 130(3):553-565. https://doi.org/10.1007/s11240-017-1246-Z 
Zhou Y, Hu L, Ye S, Jiang L, Liu S (2019b). Overexpression of an APETALA1-like gene from cucumber (Cucumis sativus L.) induces earlier flowering and abnormal leaf development in transgenic Arabidopsis. Canadian Journal of Plant Science 99:210-220. https://doi.org/10.1139/cjps-2018-0107

Zhu J, Wang X, Guo L, Xu Q, Zhao S, Li F ... Wei C (2018). Characterization and alternative splicing profiles of the lipoxygenase gene family in tea plant (Camellia sinensis). Plant and Cell Physiology 59(9):1765-1781.

OPEN ACCESS

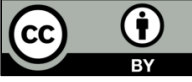

The journal offers free, immediate, and unrestricted access to peer-reviewed research and scholarly work. Users are allowed to read, download, copy, distribute, print, search, or link to the full texts of the articles, or use them for any other lawful purpose, without asking prior permission from the publisher or the author.

License - Articles published in Notulae Botanicae Horti Agrobotanici Cluj-Napoca are Open-Access, distributed under the terms and conditions of the Creative Commons Attribution (CC BY 4.0) License.

(c) Articles by the authors; UASVM, Cluj-Napoca, Romania. The journal allows the author(s) to hold the copyright/to retain publishing rights without restriction. 